\title{
How to support interest in engineering in secondary education
}

\author{
Jan Fischer, Vladimír Haasz
}

Jan Fischer, Vladimír Haasz, "How to support interest in engineering in secondary education," Proc. SPIE 11144, Photonics and Education in Measurement Science 2019, 111440E (17 September 2019); doi:

$10.1117 / 12.2528379$

SPIE Event: Joint TC1 - TC2 International Symposium on Photonics and Education in Measurement Science 2019, 2019, Jena, Germany 


\title{
How to Support Interest in Engineering in Secondary Education
}

\author{
Jan Fischer*, Vladimír Haasz \\ Czech Technical University in Prague, Faculty of Electrical Engineering, \\ Technická 2, CZ-16627 Prague 6, Czech Republic
}

\begin{abstract}
Two activities, how to increase interest in the study of electrical engineering and electronics, are described in the paper: workshops for teachers and participation in the Embedded Technology Club for students. Workshops for teachers acquaint them with the utilization of IT in a laboratory education, to make it more attractive and so to increase the interest of students to study natural sciences or engineering. The educational low-cost laboratory system and its applications in laboratories are presented during the workshop. The Embedded Technology Club (ETC) is a club intended as for students of secondary schools, who has an interest in electronics, as for other persons interested in this area. The business is focused both on education in the fundamentals of up-to-date design, development, and programming of electronic devices and solving some more simple projects concerning instrumentation. Activities are designed so that also students of secondary schools, who have no experience with electronics, but only basic knowledge in physics, can take part in. The priority is to have the interest to learn new things and to get hands-on experience.
\end{abstract}

Keywords: Secondary education, motivation, electrical engineering, laboratory education, virtual instruments, embedded technology,

\section{INTRODUCTION}

Numbers of nowadays teenagers have no interest to study natural sciences or engineering. They often prefer economics, humanities or social science, even though they commonly use different technical products in everyday life. Questions, how to motivate undergraduate students to study electrical engineering, electronics and IT, is often discussed [1], [2], [3]. A whole range of events, which can help, are organized at CTU in Prague, Faculty of electrical engineering. The most of them are focused on students and teachers of secondary schools (high schools in the USA), of course. Besides Open day, Night of scientists etc., two other types of cooperation with secondary schools are offered by the Department of Measurement:

- Workshops for secondary school teachers.

- Participation of students of secondary schools in the Embedded Technology Club (in collaboration with the Department of Telecommunication Engineering).

Nevertheless, the interest in technology could be induced already earlier. Therefore, the summer daytime camp for children is also organized. Children aged between 6 and 10 years attending the summer daytime camp have an opportunity to see, find out and also try themselves how magnetic sensors, light signals or robotics works in practice. All interactive expositions are prepared by university students. The main attraction for children is a robotic car designed by university students within the course of Laboratories of Industrial Electronics and Sensors.

\section{WORKSHOPS FOR TEACHERS}

IT education at secondary schools often concerns utilization in the administrative area only. Workshops for secondary school teachers should acquaint them with other possibilities, especially utilization of IT in a laboratory education, where students could be able to realize different experiments concerning physics, electronics, sensorics or robotics. The goal is making education more attractive and so to increase the interest of students to study natural sciences or engineering.

*fischer@fel.cvut.cz; phone +420 22435 2179; meas.fel.cvut.cz/fischer

Photonics and Education in Measurement Science 2019, edited by Maik Rosenberger,

Paul-Gerald Dittrich, Bernhard Zagar, Proc. of SPIE Vol. 11144, 111440E

(C) 2019 SPIE · CCC code: 0277-786X/19/\$21 · doi: 10.1117/12.2528379 
The educational low-cost laboratory system, which includes the kit BBC micro:bit for programming education (see figure 1a, [4]) and the platform LEO (Little Embedded Oscilloscope, see below) using the Nucleo STM32F303RE kit (see figure 1b, [5]), is presented during the workshop. Both kits utilized up-to-date 32-bit controllers with ARM Cortex M0/M4 core (more advanced platform than e.g. Arduino). The suitability of this set was verified in three runs of workshops during the last two years. Besides, both modules (BBC micro:bit and LEO with NucleoF303RE) can be lent for a short time to workshop participants free of charge to be possible to try them for education at their home school. Further, they can load new application for LEO, too.

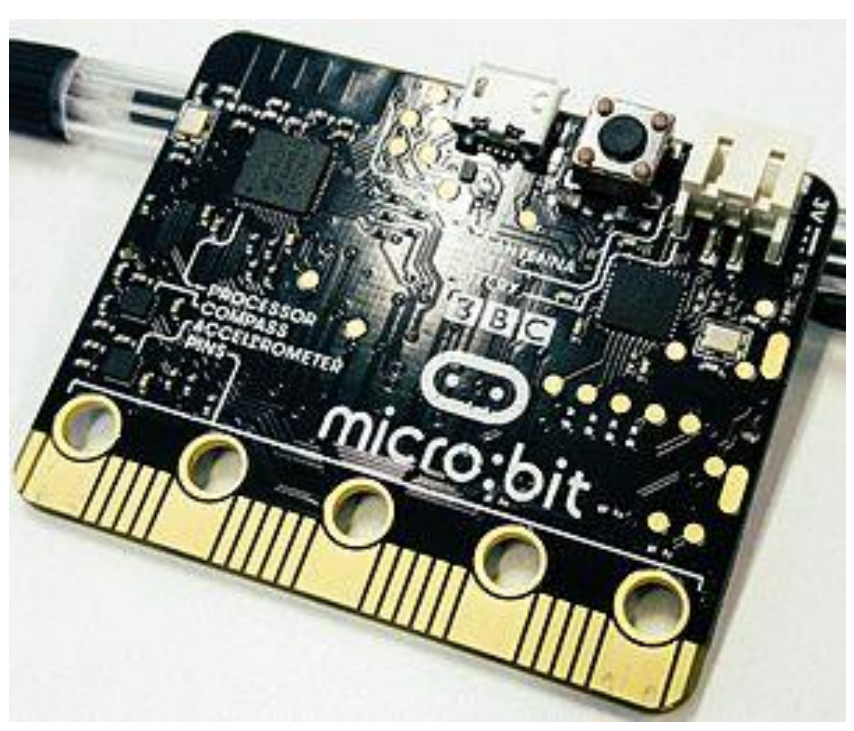

a)

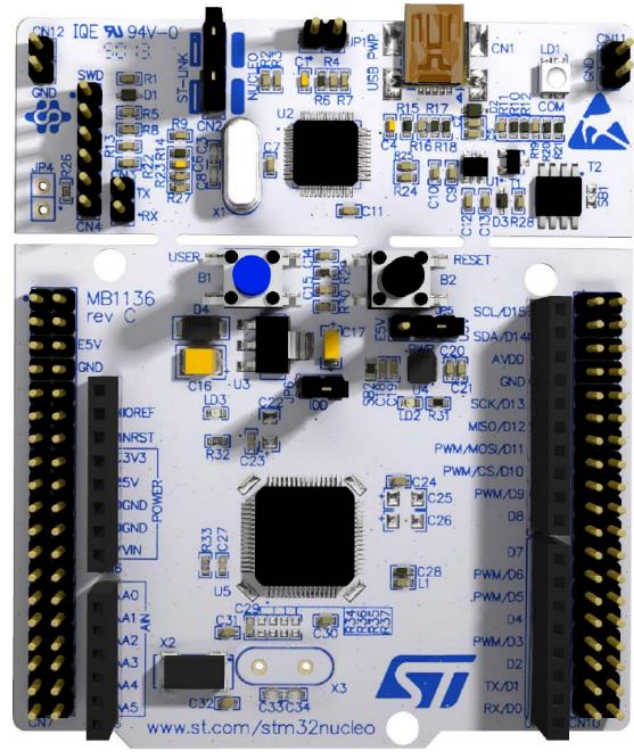

b)

Figure 1. Low-cost laboratory system, which includes the kit BBC micro:bit (a) for programming education, and the platform LEO using the Nucleo STM32F303RE kit (b)

\subsection{What is it BBC micro:bit and how to programme it?}

BBC micro:bit [4] is the kit for the first familiarisation with programming. It arose in Great Britain and it relates to BBC. However, the experience showed that the platform can be used in any further applications. The area of its utilization is not only at the primary schools but also at secondary schools after modification of contents and application of suitable programming tools. Thanks to the complex design it can be applied both for the first experiments of programming and for programming on the higher level, how it is described in [6]. The MicroPython is available and described for this purpose in the MicroPython documentation [7] and C++ in on-line IDE mbed [8]. Using C++ makes it possible to apply the programme also for other microcontrollers with ARM Cortex $M$ core used in different modules supported by mbed, in own universal development boards or even using a solderless breadboard (see below in the following section, figure 7). The platform F0-Lab with microcontroller STM32F042F6P6, which includes DC voltmeter, digitizing scope and PWM generator [9], and which was successfully used in several runs of the course on practical electronics for students entering the CTU-FEE, can be shown as an example. It can be programmed in C++ applying mbed for Nucleo STM32F042. The transferability of similar programmes designed in C++ (using mbed) among kits BBC micro:bit, NUCLEO-F042K6 and F0-Lab was verified.

\subsection{Platform LEO (Little Embedded Oscilloscope)}

A digitizing scope, a function generator, an adjustable DC voltage source, and a DC voltmeter are instruments, which are necessary for development and of electronic devices and microcontrollers applications. There are stand-alone instruments, of course, but they are not cheap or (e.g. in education at secondary school) they need not be at disposal insufficient amount. Nevertheless, all the mentioned instruments can be realized as "software-defined instrument". In this case, the powerful microcontroller STM32F303RE with its auxiliary circuits embedded in the chip is used. Only a PC and a kit Nucleo STM32F303RE (without any modification) is necessary for such measuring system realization 
Platform LEO arose at the Department of Measurement CTU-FEE within students' projects [10]. The goal of its design and development was to create a low-cost substitution of a 4-channel digitizing scope (figure 2), a two-channel function and arbitrary generator (figure 3), an adjustable DC voltage source and a DC voltmeter (figure 4). These functions should be soon completed by a universal counter to $72 \mathrm{MHz}$ for frequency, period and time interval between two signals measurement and a pulse generator. All functions are realized by software and so there is not necessary to complete the kit Nucleo F303RE (in basic function) with further circuits. A PC connected using USB is applied for results displaying.

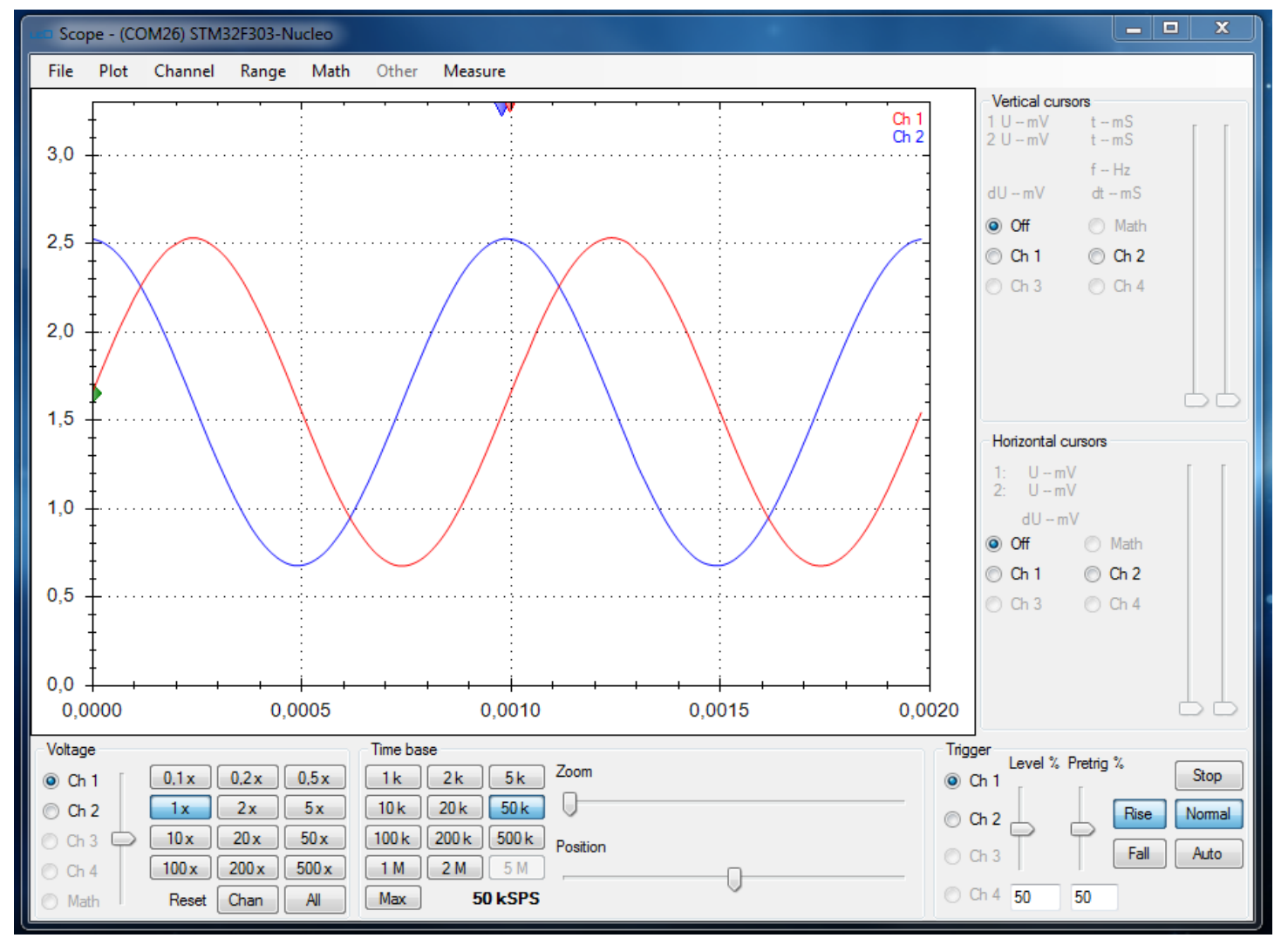

Figure 2. LEO - an oscilloscope function

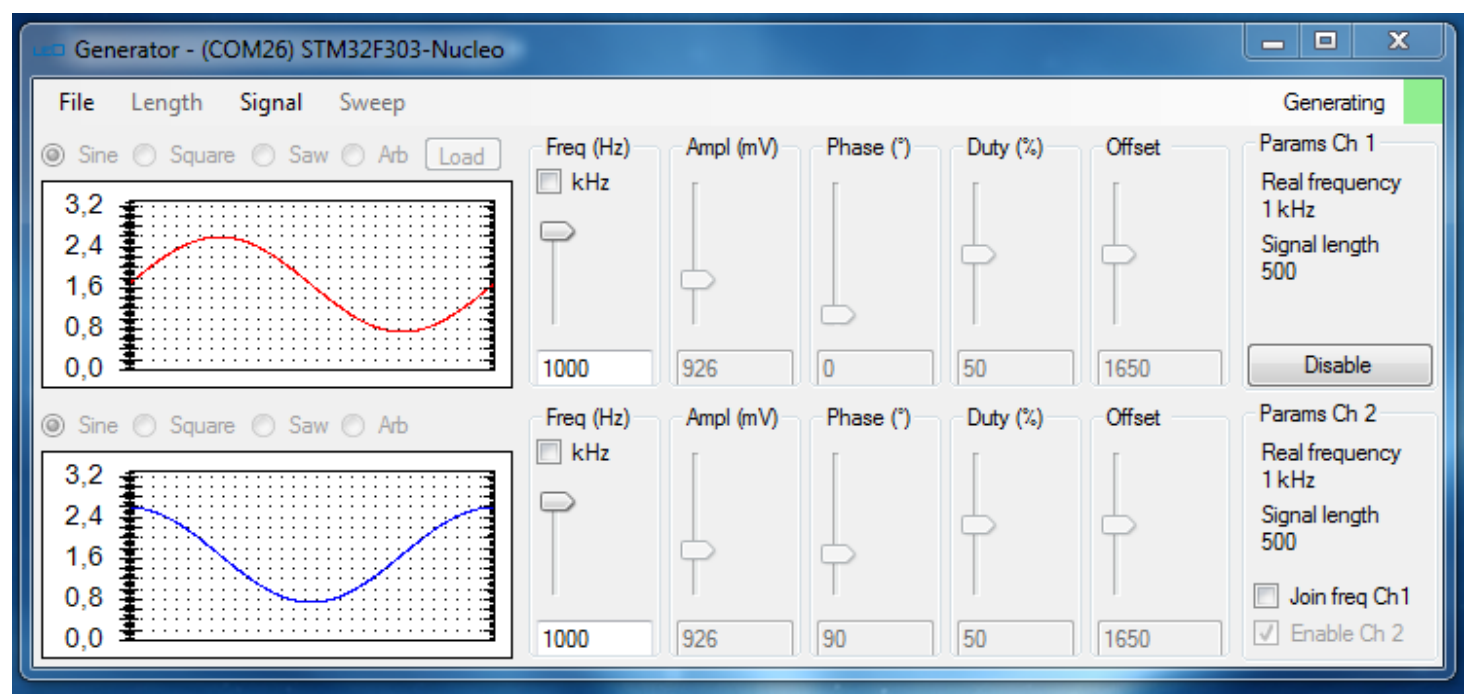

Figure 3. LEO - a function generator 


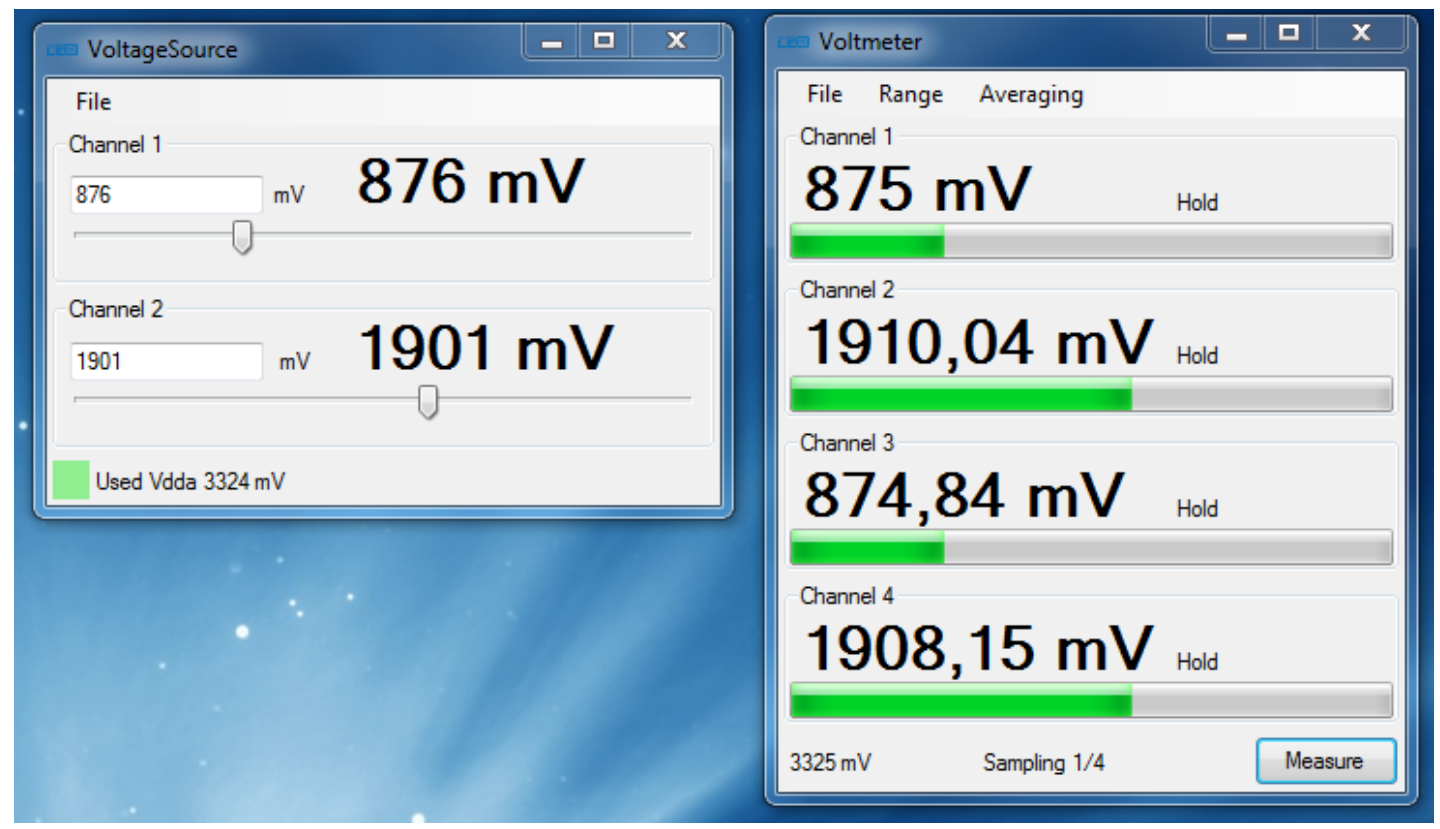

Figure 4. LEO - an adjustable DC voltage source and a voltmeter

Platform LEO can be applied not only for measurement on electrical circuits but also by education in physics, e.g. after addition of simple optical sensors for speed measurement, measurement of frequency or period of vibrations etc. Moreover, the kit Nucleo F303RE can be applied as a separate microcontroller for education in programming using online IDE mbed, too. The platform LEO was successfully exhibited on the Embedded World 2018 (figure 5).

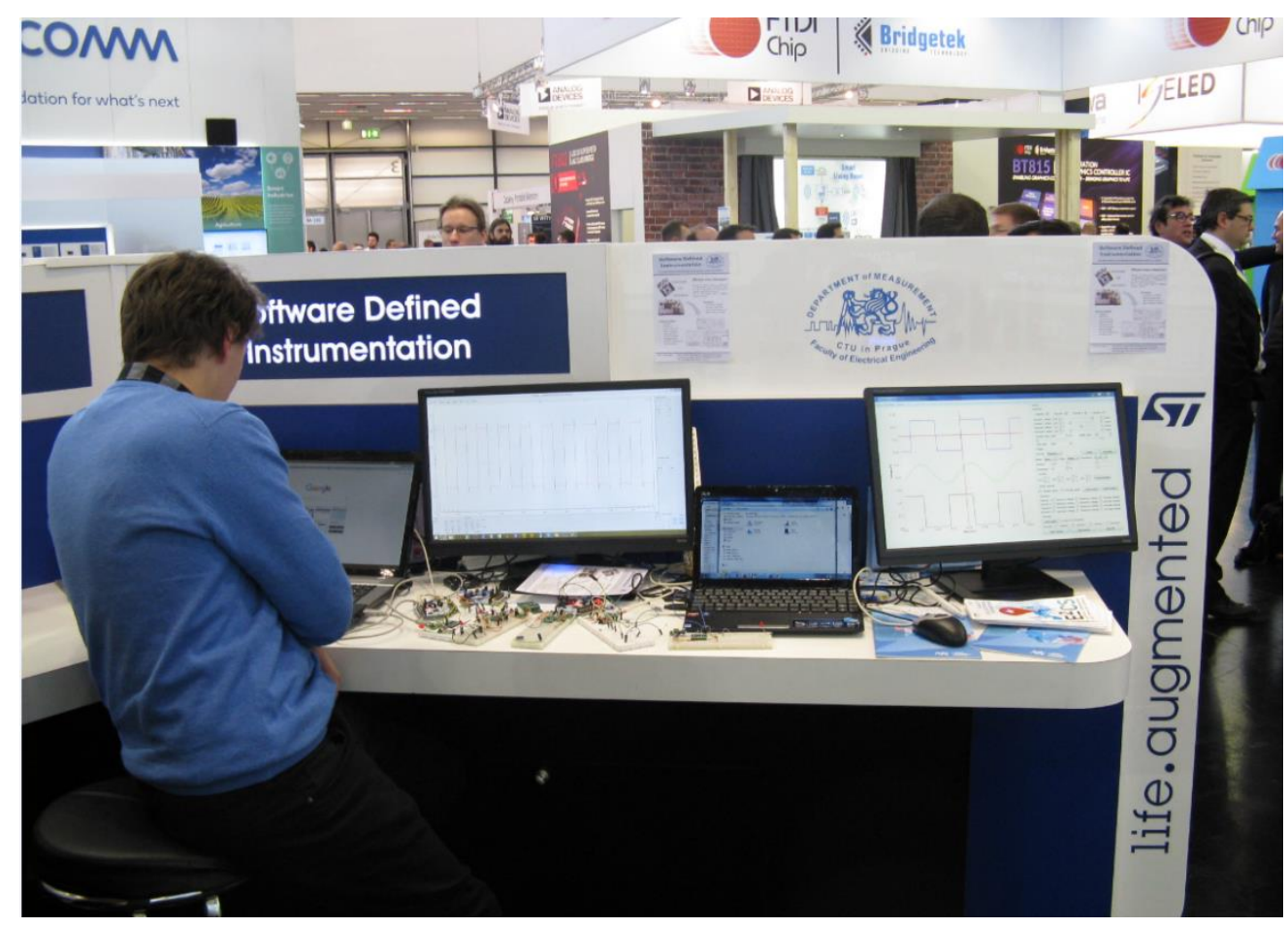

Figure 5. LEO - Embedded World 2018 


\section{EMBEDDED TECHNOLOGY CLUB}

The Embedded Technology Club (ETC) is a club intended as for students of secondary schools, who has an interest in electronics, as for other persons interested in this area. This idea was inspired e.g. by [11]. The business of ETC is focused both on education in the fundamentals of up-to-date design, development, and programming of electronic devices and to solving some more simple projects concerning measurement and instrumentation. Activities are designed so that also students of secondary schools, who have no experience with electronics, but only basic knowledge in physics, can take part in. The priority is to have the interest to learn new things and to get hands-on experience. The ETC is open during each semester one evening in the week.

A part of the time spent in ETC is dedicated to lectures, which are specified especially for new participants from secondary schools. So, they gain basic knowledge, which is later necessary for solving individual projects. It concerns the fundamentals of electronics and digital technology essential for the realization of embedded systems using up-to-date 32-bit microcontrollers with the ARM Cortex-M core. For their programming, the online IDE mbed is applied in ETC by experiments and practical realizations. Each participant should be able after this teaching part to design and develop a small system using microcontroller STM32F042F6P6. It could be e.g. a simple measuring instrument, speed control of DC engine, robotic plaything, digital temperature controller etc. All these simple projects are at first realized using a solderless breadboard (see figure 6). Experienced participants solve later more complicated tasks, e.g. a reaction tester, an approaching sensor, etc. (see below).

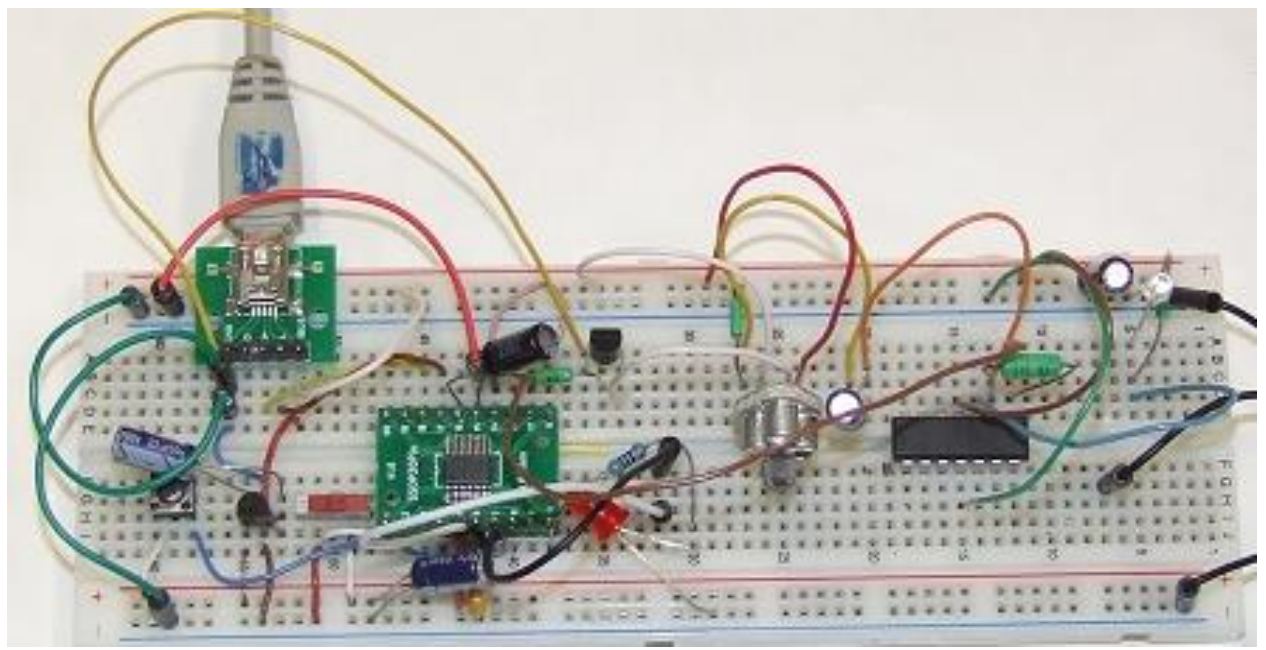

Figure 6. An example of realization using the solderless breadboard (left: STM32F042 with USB connection realizing a simple oscilloscope, right: a circuit with an infrared LED and phototransistors)

The reaction tester is controlled by microcontroller STM32F042, which activates (in the simplest variant) randomly a LED and a tested person should press the button in a set time limit. The more complicated two channels variant includes two LEDs and two buttons, independently for left and right hand. The following extension makes it possible to communicate with PC using the USB interface to register and to process test results.

Another task concerns an approaching sensor. This is also controlled by microcontroller STM32F042 and it is based on an optoelectronic reflex sensor. Its hardware is very simple (see figure 7). It uses the infrared emitting diode IR, which is supplied by the modulated current generated by the microcontroller MC, and the phototransistor IPT with an infrared filter, which partly suppress daylight. A part of radiation reflected off an obstacle is detected by the phototransistor IPT. If the voltage $u_{\text {sing }}$ reaches a set threshold, the obstacle is signalized. Digitalization of the voltage $u_{\text {sig }}$, which depends on the distance from the obstacle, and the following digital signal processing is executed by the microcontroller and the corresponding software. The "lock-in" method is used to reach enough sensitivity. The device can be extended of an acoustic signalization, where the pitch of a tone corresponds to the distance. 


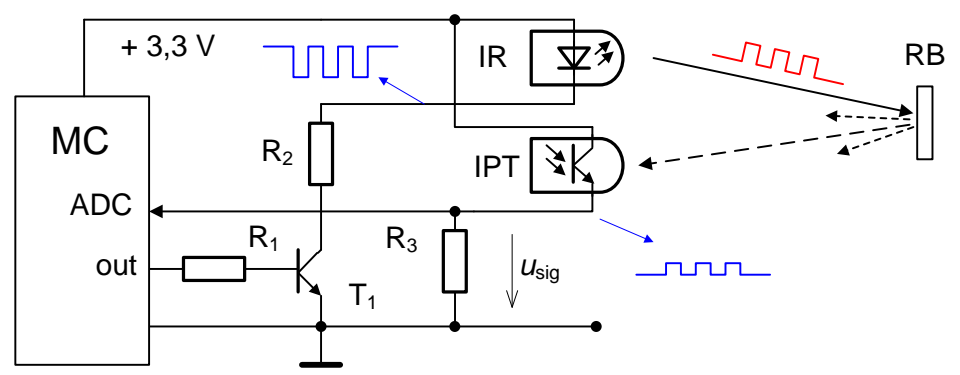

Figure 7. The connection of the approaching sensor

\section{CONCLUSION}

Both activities mention above (workshops for secondary school teachers and ETC for students) should contribute increasing of interest in study engineering after finishing a secondary school. We believe that an attractive education using up-to-date tools concerning instrumentation can help with it. Therefore, the workshops, which show how to use it, were organized for secondary school teachers. Besides, the ETC makes it possible to familiarise students of secondary schools with this approach and to teach them how to design, develop and programme simple electronics devices.

\section{REFERENCES}

[1] Mamaril, N. A., Usher, E. L., Economy, D. R., Kennedy, M. S., "An examination of students' motivation in engineering service courses," Proc. of 2013 IEEE Frontiers in Education Conference, 1825-1827 (2013).

[2] Foley, J. M., Daly, S., Lenaway, C., Phillips, J., "Investigating Student Motivation and Performance in Electrical Engineering and Its Subdisciplines," IEEE Transactions on Education 59(4), 241-247 (2016).

[3] Kori, K., Pedaste, M., Altin, H., Tõnisson, E., Palts, T., "Factors That Influence Students' Motivation to Start and to Continue Studying Information Technology in Estonia," IEEE Transactions on Education, 59(4), 255262, (2016).

[4] Arm Limited, "BBC micro:bit," Arm Limited, 2019, https://os.mbed.com/platforms/Microbit/ (22 July 2019).

[5] Arm Limited, "NUCLEO-F303RE," Arm Limited, 2019, https://os.mbed.com/platforms/ST-Nucleo-F303RE/ (22 July 2019).

[6] BBC Micro:bit, "Power your imagination with code," Micro:bit Educational Foundation 2019, https://www.microbit.co.uk/create-code\# (22 July 2019).

[7] Multiply authors, "BBC micro:bit MicroPython documentation, ” BBC Micro:bit 2016, https://microbit-micropython.readthedocs.io/en/latest/ (22 July 2019).

[8] Arm Limited, "Mbed OS5," Arm Limited 2019, https://os.mbed.com/, (22 July 2019).

[9] STMicroelectronics, "STM32F042K6," STMicroelectronics, 2019, https://www.st.com/en/microcontrollers/stm32f042k6.html, (22 July 2019)..

[10] LEO Team, "Little Embedded Oscilloscope," CTU-FEE, Dept. of Measurement 2018, https://leo.fel.cvut.cz/en (22 July 2019).

[11]Braun, G., "Making Things Real in Electronics Laboratories," Proc. of Frontiers in Education Conference 1995, Vol. 2, 4c2.10 - 4c2.13 (1995)

\section{ACKNOWLEDGMENT}

The activities mentioned above are supported by the companies $2 \mathrm{~N}$ Telekomunikace a.s. and STMicroelectronics. 